\title{
ESSENTIAL NORM OF EXTENDED CESÀRO OPERATORS FROM ONE BERGMAN SPACE TO ANOTHER
}

\author{
ZHANGJIAN HU
}

(Received 20 May 2011)

\section{Abstract}

Let $A^{p}(\varphi)$ be the $p$ th Bergman space consisting of all holomorphic functions $f$ on the unit ball $B$ of $\mathbb{C}^{n}$ for which $\|f\|_{p, \varphi}^{p}=\int_{B}|f(z)|^{p} \varphi(z) d A(z)<+\infty$, where $\varphi$ is a given normal weight. Let $T_{g}$ be the extended Cesàro operator with holomorphic symbol $g$. The essential norm of $T_{g}$ as an operator from $A^{p}(\varphi)$ to $A^{q}(\varphi)$ is denoted by $\left\|T_{g}\right\|_{e, A^{p}(\varphi) \rightarrow A^{q}(\varphi)}$. In this paper it is proved that, for $p \leq q$,

$$
\left\|T_{g}\right\|_{e, A^{p}(\varphi) \rightarrow A^{q}(\varphi)} \simeq \limsup _{|z| \rightarrow 1}|\mathfrak{R} g(z)|\left(\frac{\left(1-|z|^{2}\right)^{k-(n+1)}}{\varphi(z)}\right)^{1 / k}
$$

with $1 / k=(1 / p)-(1 / q)$, where $\mathfrak{R} g(z)$ is the radial derivative of $g$; and for $p>q$,

$$
\left\|T_{g}\right\|_{e, A^{p}(\varphi) \rightarrow A^{q}(\varphi)}=\lim _{r \rightarrow 1} \int_{|z| \geq r}|g(z)-g(0)|^{s} \varphi(z) d A(z)
$$

with $1 / s=(1 / q)-(1 / p)$.

2010 Mathematics subject classification: primary 32A37; secondary 47B38.

Keywords and phrases: Bergman spaces, extended Cesàro operators, essential norm.

\section{Introduction}

Let $B$ be the unit ball of $\mathbb{C}^{n}$; if $n=1$ then the unit disc is also denoted by $D$. Let $d A$ be the Lebesgue volume measure on $B$ and let $d \sigma$ be the normalised surface measure on $\partial B$. Write $\beta(\cdot, \cdot)$ for the Bergman distance on $B$. Given $z \in B$ and $r>0$, the Bergman ball with centre $z$ and radius $r$ is $E(z, r)=\{w \in B: \beta(z, w)<r\}$. Let $H(B)$ be the family of all holomorphic functions on $B$. A positive continuous function $\varphi$ on $[0,1)$ is called normal if there are two constants $b>a>-1$ such that

$$
\frac{\varphi(r)}{(1-r)^{a}} \downarrow 0, \quad \frac{\varphi(r)}{(1-r)^{b}} \uparrow \infty
$$

This research is partially supported by the National Natural Science Foundation of China (10771064), Natural Science Foundation of Zhejiang province (Y7080197, Y6090036, Y6100219) and Foundation of Creative Group in Universities of Zhejiang Province (T200924).

(c) 2011 Australian Mathematical Publishing Association Inc. 0004-9727/2011 \$16.00 
as $r \rightarrow 1^{-}$. If $\varphi$ is normal, then we extend it to $B$ by $\varphi(z)=\varphi(|z|)$. For $0<p<\infty$, the weighted Bergman space $A^{p}(\varphi)$ consists of all functions $f \in H(B)$ for which

$$
\|f\|_{p, \varphi}^{p}=\int_{B}|f(z)|^{p} \varphi(z) d A(z)<+\infty .
$$

For $g \in H(B)$, with symbol $g$, the extended Cesàro operator $T_{g}$ is defined on $H(B)$ as

$$
T_{g}(f)(z)=\int_{0}^{1} f(t z) \Re g(t z) \frac{d t}{t}, \quad f \in H(B), z \in B,
$$

where $\mathfrak{R} g(z)=\sum_{j=1}^{n} z_{j}\left(\partial g / \partial z_{j}\right)$ is the radial derivative of $g$, as in [Ru80].

Let $X$ and $Y$ be two Banach (or Fréchet) spaces, and let $T$ be a linear operator from $X$ to $Y$ with the operator norm $\|T\|_{X \rightarrow Y}$. Let $K$ be the set of all compact linear operators from $X$ to $Y$. The essential norm of $T$, denoted by $\|T\|_{e, X \rightarrow Y}$, is defined as

$$
\|T\|_{e, X \rightarrow Y}=\inf _{Q \in K}\|T-Q\|_{X \rightarrow Y} .
$$

The operator $T_{g}$ in one variable was studied in [AC01, AS95, AS97]. In the higher-dimensional case, it was first studied in [Hu03, Hu04], where the boundedness and compactness on Bergman spaces (or mixed norm spaces) were completely characterised. Recently, in [HT10], Schatten(-Herz) class extended Cesàro operators on $A^{2}(\varphi)$ were considered. The purpose of this note is to study the essential norm for $T_{g}$ as an operator from $A^{p}(\varphi)$ to $A^{q}(\varphi)$ for all possible $0<p, q<\infty$. Some of our results in the one-variable case with $p \leq q$ were obtained in [Ra07].

In what follows, we use $C$ to denote a positive constant whose value may change from line to line but does not depend on the functions in $H(B)$. The expression ' $A \simeq B$ ' means there exists some $C$ such that $C^{-1} A \leq B \leq C A$.

\section{Main theorem}

Given $g \in H(B)$, write $M_{\infty}(g, r)=\sup _{|z|=r}|g(z)|$. It is well known that $M_{\infty}(g, r)$ is increasing with $r$. In the proof of our main theorem, we need the following lemma, which is of independent interest.

LeMma 2.1. Let $\psi$ be a positive continuous function on the interval $[0,1)$ with $0<$ $\lim \sup _{r \rightarrow 1} \psi(r) \leq \infty$. Then there is some constant $C$ such that, for all $g \in H(B)$,

$$
\sup _{z \in B}|g(z)| \psi(|z|) \leq C \limsup _{|z| \rightarrow 1}|g(z)| \psi(|z|) .
$$

Proof. First we prove that there exist a constant $C$ and a sequence $\left\{r_{j}\right\}, r_{j} \rightarrow 1$ as $j \rightarrow \infty$, such that

$$
\sup _{0 \leq \rho<r_{j}} \psi(\rho) \leq C \psi\left(r_{j}\right) .
$$

In fact, if $0<\limsup _{r \rightarrow 1} \psi(r)<\infty$, then we can pick some sequence $\left\{r_{j}\right\}$ such that $r_{j} \rightarrow 1$ as $j \rightarrow \infty$ and $\psi\left(r_{j}\right) \geq \frac{1}{2} \lim \sup _{r \rightarrow 1} \psi(r)$. Hence

$$
\sup _{0 \leq \rho<r_{j}} \psi(\rho) \leq \sup _{0 \leq \rho<1} \psi(\rho) \leq \frac{2 \sup _{0 \leq \rho<1} \psi(\rho)}{\lim \sup _{r \rightarrow 1} \psi(r)} \psi\left(r_{j}\right)=C \psi\left(r_{j}\right) .
$$


If $\lim \sup _{r \rightarrow 1} \psi(r)=\infty$, then we can take some $r_{j} \rightarrow 1$ so that

$$
\sup _{0 \leq \rho \leq r_{j}} \psi(\rho)=\psi\left(r_{j}\right)
$$

Otherwise, we would have some $r_{0}$ such that, for all $r \in\left[r_{0}, 1\right)$,

$$
\sup _{0 \leq \rho \leq r} \psi(\rho)>\psi(r)
$$

Then $\sup _{0 \leq \rho \leq r} \psi(\rho)$ cannot be achieved at any point in $\left[r_{0}, r\right]$. Hence lim $\sup _{r \rightarrow 1} \psi(r) \leq$ $\sup _{0 \leq \rho \leq r_{0}} \psi(\rho)$, a contradiction. From (2.3) and (2.4), (2.2) follows.

For $g \in H(B)$, we claim that there is some $\eta=\eta(g) \in(0,1)$ such that

$$
M_{\infty}(g, r) \psi(r) \leq 2 \limsup _{r \rightarrow 1} M_{\infty}(g, r) \psi(r)
$$

for all $\eta \leq r<1$. In fact, if $\lim _{r \rightarrow 1} M_{\infty}(g, r) \psi(r)=0$, then $\lim _{r \rightarrow 1} M_{\infty}(g, r)=0$ by the hypothesis $\lim \sup _{r \rightarrow 1} \psi(r)>0$. This means that $g$ is identically zero. Hence (2.5) is valid for all $\eta \in[0,1)$. If $\lim _{r \rightarrow 1} M_{\infty}(g, r) \psi(r)>0$, the estimate (2.5) is valid for all $\eta$ sufficiently near 1 by the definition of lim sup.

Now, for any $g \in H(B)$, fix some $r_{j}$ satisfying (2.2) such that $r_{j} \in[\eta(g), 1)$. Then, by (2.5),

$$
\begin{aligned}
\sup _{0 \leq r<1} M_{\infty}(g, r) \psi(r) & \leq \sup _{0 \leq r \leq r_{j}} M_{\infty}(g, r) \psi(r)+\sup _{r_{j} \leq r<1} M_{\infty}(g, r) \psi(r) \\
& \leq M_{\infty}\left(g, r_{j}\right) \sup _{0 \leq r \leq r_{j}} \psi(r)+2 \underset{r \rightarrow 1}{\lim \sup } M_{\infty}(g, r) \psi(r) \\
& \leq C M_{\infty}\left(g, r_{j}\right) \psi\left(r_{j}\right)+2 \underset{r \rightarrow 1}{\lim \sup } M_{\infty}(g, r) \psi(r) \\
& \leq C \limsup _{r \rightarrow 1} M_{\infty}(g, r) \psi(r),
\end{aligned}
$$

where the constant $C$ is independent of $g \in H(B)$. The estimate (2.1) follows.

Lemma 2.2. Suppose that $g \in H(B)$. Then, for $0<p \leq q<\infty$,

$$
\left\|T_{g}\right\|_{A^{p}(\varphi) \rightarrow A^{q}(\varphi)} \simeq \sup _{z \in B}|\mathfrak{R} g(z)|\left(\frac{\left(1-|z|^{2}\right)^{k-(n+1)}}{\varphi(z)}\right)^{1 / k}
$$

with $1 / k=(1 / p)-(1 / q)$; and, for $0<q<p<\infty$,

$$
\left\|T_{g}\right\|_{A^{p}(\varphi) \rightarrow A^{q}(\varphi)} \simeq\|g-g(0)\|_{s, \varphi}
$$

with $1 / s=(1 / q)-(1 / p)$.

See [Hu04, Theorem 5]. Things to pay attention to are that, as pointed out in [Hu04, Remark 2], normality here is the same as that defined by conditions $\left(P_{1}\right)$ and $\left(P_{2}\right)$ in [AS97, Hu04] in the sense that they induce the same $p$ th Bergman space with equivalent norms. Also, we have $\varphi^{*} \simeq \varphi$, where $\varphi^{*}(r)=(1 /(1-r)) \int_{e}^{(1+r) / 2} \varphi(t) d t$, as in [Hu04]. 
Theorem 2.3. Let $g \in H(B)$. Then, for $0<p \leq q<\infty$,

$$
\left\|T_{g}\right\|_{e, A^{p}(\varphi) \rightarrow A^{q}(\varphi)} \simeq \limsup _{|z| \rightarrow 1}|\mathfrak{R} g(z)|\left(\frac{\left(1-|z|^{2}\right)^{k-(n+1)}}{\varphi(z)}\right)^{1 / k}
$$

with $1 / k=(1 / p)-(1 / q)$; and, for $p>q$,

$$
\left\|T_{g}\right\|_{e, A^{p}(\varphi) \rightarrow A^{q}(\varphi)}=\lim _{|z| \rightarrow 1} \int_{|z| \geq r}|g(z)-g(0)|^{s} \varphi(z) d A(z)
$$

with $1 / s=(1 / q)-(1 / p)$.

Proof. We suppose first that $0<p \leq q<\infty$. Given $\zeta \in B$, let the function $f_{\zeta}$ be

$$
f_{\zeta}(z)=\left(\frac{\left(1-|\zeta|^{2}\right)^{\beta}}{\varphi(\zeta)(1-\langle z, \zeta\rangle)^{n+1+\beta}}\right)^{1 / p},
$$

where $\beta>b$ is fixed with $b$ as in (1.1). As indicated in [Hu04, proof of Theorem 2],

$$
\left\|f_{\zeta}\right\|_{p, \varphi} \leq C \quad \text { and } \quad f_{\zeta}(\zeta)=\frac{1}{\left(\varphi(\zeta)\left(1-|\zeta|^{2}\right)^{n+1}\right)^{1 / p}} .
$$

Further, it is easy to check that $f_{\zeta}(z)$ goes to 0 uniformly on any compact subset of $B$ as $|\zeta| \rightarrow 1$. Hence, for each $Q \in K$,

$$
\lim _{|\zeta| \rightarrow \infty}\left\|Q f_{\zeta}\right\|_{q, \varphi}=0 .
$$

Let $\zeta_{j} \in B$ be such that

$$
\lim _{j \rightarrow \infty}\left|\mathfrak{R} g\left(\zeta_{j}\right)\right|\left(\frac{\left(1-\left|\zeta_{j}\right|^{2}\right)^{k-(n+1)}}{\varphi\left(\zeta_{j}\right)}\right)^{1 / k}=\limsup _{|z| \rightarrow 1}|\mathfrak{R} g(z)|\left(\frac{\left(1-|z|^{2}\right)^{k-(n+1)}}{\varphi(z)}\right)^{1 / k} .
$$

Notice that $\mathfrak{R}\left(T_{g} f\right)=f \mathfrak{R} g$. Then, for $Q \in K$, by [Hu04, Theorem 1],

$$
\begin{aligned}
\left\|T_{g}-Q\right\|_{A^{p}(\varphi) \rightarrow A^{q}(\varphi)} & \geq C \limsup _{j \rightarrow \infty}\left\|\left(T_{g}-Q\right) f_{\zeta_{j}}\right\|_{q, \varphi} \\
& \geq C\left(\limsup _{j \rightarrow \infty}\left\|T_{g} f_{\zeta_{j}}\right\|_{q, \varphi}-\lim _{j \rightarrow \infty}\left\|Q f_{\zeta_{j}}\right\|_{q, \varphi}\right) \\
& =C \limsup _{j \rightarrow \infty}\left\|T_{g} f_{\zeta_{j}}\right\|_{q, \varphi} \\
& \simeq C \limsup _{j \rightarrow \infty}\left\|\mathfrak{R}\left(T_{g} f_{\zeta_{j}}\right)(z)\left(1-|z|^{2}\right)\right\|_{q, \varphi} \\
& =C \limsup _{j \rightarrow \infty}\left(\int_{B}\left|f_{\zeta_{j}}(z) \mathfrak{R} g(z)\left(1-|z|^{2}\right)\right|^{q} \varphi(z) d A(z)\right)^{1 / q} \\
& \geq C \limsup _{j \rightarrow \infty}\left(\int_{E\left(\zeta_{j} r\right)}\left|f_{\zeta_{j}}(z) \mathfrak{R} g(z)\left(1-|z|^{2}\right)\right|^{q} \varphi(z) d A(z)\right)^{1 / q} \\
& \geq C \limsup _{j \rightarrow \infty}\left(\left|f_{\zeta_{j}}\left(\zeta_{j}\right) \mathfrak{R} g\left(\zeta_{j}\right)\right|^{q}\left(1-\left|\zeta_{j}\right|^{2}\right)^{q+(n+1)} \varphi\left(\zeta_{j}\right)\right)^{1 / q} \\
& =C \limsup _{j \rightarrow \infty} \mid \mathfrak{R} g\left(\zeta_{j}\right)\left(\frac{\left(1-\left|\zeta_{j}\right|^{2}\right)^{k-(n+1)}}{\varphi\left(\zeta_{j}\right)}\right)^{1 / k} \cdot
\end{aligned}
$$


By the definition of essential norm and the estimate above, we know that

$$
\left\|T_{g}\right\|_{e, A^{p}(\varphi) \rightarrow A^{q}(\varphi)} \geq C \limsup _{|z| \rightarrow 1}|\mathfrak{R} g(z)|\left(\frac{\left(1-|z|^{2}\right)^{k-(n+1)}}{\varphi(z)}\right)^{1 / k} .
$$

We now prove the reverse inequality. This will be split into two cases. First, let

$$
\limsup _{r \rightarrow 1}\left(\frac{\left(1-|z|^{2}\right)^{k-(n+1)}}{\varphi(z)}\right)^{1 / k}=0 .
$$

We may suppose that $g \in H(B)$ satisfies

$$
\limsup _{|z| \rightarrow 1}|\mathfrak{R} g(z)|\left(\frac{\left(1-|z|^{2}\right)^{k-(n+1)}}{\varphi(z)}\right)^{1 / k}<\infty .
$$

By (1.1), there is some positive constant $\alpha$ such that

$$
\sup _{z \in B}|\mathfrak{R} g(z)|\left(1-|z|^{2}\right)^{\alpha}<\infty .
$$

Hence [Zh05, Theorem 2.7] tells us that

$$
\mathfrak{R} g(z)=c_{\alpha} \int_{B} \frac{\mathfrak{R} g(w)}{(1-\langle z, w\rangle)^{n+1+\alpha}}\left(1-|w|^{2}\right)^{\alpha} d A(w),
$$

with $c_{\alpha}$ a fixed constant depending on $n$ and $\alpha$. For $0<\rho<1$, define $G_{\rho}$ by

$$
G_{\rho}(z)=c_{\alpha} \int_{B} \frac{\mathfrak{R} g(w)}{(1-\langle z, w\rangle)^{n+1+\alpha}} \chi_{\rho}(w)\left(1-|w|^{2}\right)^{\alpha} d A(w),
$$

where

$$
\chi_{\rho}(w)= \begin{cases}1 & \text { if }|w| \leq \rho, \\ 0 & \text { if } \rho<|w|<1 .\end{cases}
$$

It is trivial to verify that $G_{\rho}(z)$ is holomorphic on the closed unit ball $\bar{B}$, and also $G_{\rho}(0)=0$ since $\mathfrak{R} g(0)=0$. Set $g_{\rho}(z)=\int_{0}^{1}\left(G_{\rho}(t z) / t\right) d t$; then $g_{\rho}$ is also holomorphic on $\bar{B}$, and

$$
\mathfrak{R} g_{\rho}(z)=G_{\rho}(z)
$$

Hence, using (2.9),

$$
\lim _{|z| \rightarrow 1}\left|\mathfrak{R} g_{\rho}(z)\right|\left(\frac{\left(1-|z|^{2}\right)^{k-(n+1)}}{\varphi(z)}\right)^{1 / k}=0 .
$$

Theorem 6 in [Hu04] tells us that $T_{g_{\rho}}$ is compact from $A^{p}(\varphi)$ to $A^{q}(\varphi)$. Therefore, by Lemma 2.2 and (2.10), (2.11), (2.12),

$$
\begin{aligned}
\left\|T_{g}\right\|_{e, A^{p}(\varphi) \rightarrow A^{q}(\varphi)} & \leq\left\|T_{g}-T_{g_{\rho}}\right\| \\
& \leq C \sup _{z \in B}\left|\mathfrak{R} g(z)-\mathfrak{R} g_{\rho}(z)\right|\left(\frac{\left(1-|z|^{2}\right)^{k-(n+1)}}{\varphi(z)}\right)^{1 / k}
\end{aligned}
$$




$$
\begin{aligned}
& =C \sup _{z \in B}\left(\frac{\left(1-|z|^{2}\right)^{k-(n+1)}}{\varphi(z)}\right)^{1 / k}\left|\int_{|w| \geq \rho} \frac{\mathfrak{R} g(w)}{(1-\langle z, w\rangle)^{n+1+\alpha}}\left(1-|w|^{2}\right)^{\alpha} d A(w)\right| \\
& \leq C \sup _{|w| \geq \rho}|\mathfrak{R} g(w)|\left(\frac{\left(1-|w|^{2}\right)^{k-(n+1)}}{\varphi(w)}\right)^{1 / k} \sup _{z \in B}\left(\frac{\left(1-|z|^{2}\right)^{k-(n+1)}}{\varphi(z)}\right)^{1 / k} \\
& \quad \times \int_{B}\left(\frac{\varphi(z)}{\left(1-|w|^{2}\right)^{k-(n+1)}}\right)^{1 / k} \frac{\left(1-|w|^{2}\right)^{\alpha}}{|1-\langle z, w\rangle|^{n+1+\alpha}} d A(w) .
\end{aligned}
$$

Using the approach in [Hu03, proof of Lemma 2],

$$
\int_{0}^{1} \frac{\left(1-|t|^{2}\right)^{\alpha-1+((n+1) / k)}}{(1-t|z|)^{1+\alpha}}(\varphi(t))^{1 / k} d t \leq C\left(\frac{\varphi(z)}{\left(1-|z|^{2}\right)^{k-(n+1)}}\right)^{1 / k} .
$$

Therefore, by [Ru80, Proposition 1.4.10],

$$
\begin{aligned}
& \left\|T_{g}\right\|_{e, A^{p}(\varphi) \rightarrow A^{q}(\varphi)} \\
& \leq C \sup _{|w| \geq \rho}|\mathfrak{R} g(w)|\left(\frac{\left(1-|w|^{2}\right)^{k-(n+1)}}{\varphi(w)}\right)^{1 / k} \\
& \times \sup _{z \in B}\left(\frac{\left(1-|z|^{2}\right)^{k-(n+1)}}{\varphi(z)}\right)^{1 / k} \int_{0}^{1} d t \int_{\partial B} \frac{\left(1-|t|^{2}\right)^{\alpha-1+((n+1) / k)}}{|1-t\langle z, \zeta\rangle|^{n+1+\alpha}}(\varphi(t))^{1 / k} d \sigma(\zeta) \\
& \leq C \sup _{|w| \geq \rho}|\Re g(w)|\left(\frac{\left(1-|w|^{2}\right)^{k-(n+1)}}{\varphi(w)}\right)^{1 / k} \\
& \times \sup _{z \in B}\left(\frac{\left(1-|z|^{2}\right)^{k-(n+1)}}{\varphi(z)}\right)^{1 / k} \int_{0}^{1} \frac{\left(1-|t|^{2}\right)^{\alpha-1+((n+1) / k)}}{(1-t|z|)^{1+\alpha}}(\varphi(t))^{1 / k} d t \\
& \leq C \sup _{|w| \geq \rho}|\mathfrak{R} g(w)|\left(\frac{\left(1-|w|^{2}\right)^{k-(n+1)}}{\varphi(w)}\right)^{1 / k} .
\end{aligned}
$$

This implies that

$$
\left\|T_{g}\right\|_{e, A^{p}(\varphi) \rightarrow A^{q}(\varphi)} \leq C \limsup _{|z| \rightarrow 1}|\Re g(z)|\left(\frac{\left(1-|z|^{2}\right)^{k-(n+1)}}{\varphi(z)}\right)^{1 / k} .
$$

For the case

$$
\limsup _{r \rightarrow 1}\left(\frac{\left(1-|z|^{2}\right)^{k-(n+1)}}{\varphi(z)}\right)^{1 / k} \neq 0
$$

by Lemmas 2.1 and 2.2 we have

$$
\begin{aligned}
\left\|T_{g}\right\|_{e, A^{p}(\varphi) \rightarrow A^{q}(\varphi)} & \leq\left\|T_{g}\right\| \\
& \simeq \sup _{0 \leq r<1} M_{\infty}(\mathfrak{R} g, r)\left(\frac{\left(1-r^{2}\right)^{k-(n+1)}}{\varphi(r)}\right)^{1 / k} \\
& \leq C \limsup _{r \rightarrow 1} M_{\infty}(\mathfrak{R} g, r)\left(\frac{\left(1-r^{2}\right)^{k-(n+1)}}{\varphi(r)}\right)^{1 / k} .
\end{aligned}
$$

The estimates (2.6) come from (2.8) and (2.13), (2.14). 
We now suppose that $0<q<p<\infty$. Let $s>0$ be such that $1 / s=(1 / q)-(1 / p)$. If $\|g-g(0)\|_{s, \varphi}<\infty$, by [Hu04, Theorem 6] $T_{g}$ is itself compact from $A^{p}(\varphi)$ to $A^{q}(\varphi)$. Notice that $\|g-g(0)\|_{s, \varphi}<\infty$ implies that

$$
\lim _{r \rightarrow 1} \int_{|z| \geq r}|g(z)-g(0)|^{s} \varphi(z) d A(z)=0 .
$$

Hence

$$
\left\|T_{g}\right\|_{e, A^{p}(\varphi) \rightarrow A^{q}(\varphi)}=0=\lim _{r \rightarrow 1} \int_{|z| \geq r}|g(z)-g(0)|^{s} \varphi(z) d A(z) .
$$

On the other hand, if $\|g-g(0)\|_{s, \varphi}=\infty$, then, for each $r \in[0,1)$,

$$
\int_{|z| \geq r}|g(z)-g(0)|^{s} \varphi(z) d A(z)=\infty .
$$

Theorem 5 in [Hu04] tells us that $T_{g}$ is not bounded from $A^{p}(\varphi)$ to $A^{q}(\varphi)$. Hence, for each compact operator $Q,\left\|T_{g}-Q\right\|_{A^{p}(\varphi) \rightarrow A^{q}(\varphi)}=\infty$. Therefore,

$$
\left\|T_{g}\right\|_{e, A^{p}(\varphi) \rightarrow A^{q}(\varphi)}=\infty=\lim _{r \rightarrow 1} \int_{|z| \geq r}|g(z)-g(0)|^{s} \varphi(z) d A(z) .
$$

The estimate (2.7) follows from (2.15) and (2.16). The proof is complete.

REMARK 2.4. The case in which

$$
\limsup _{r \rightarrow 1}\left(\frac{\left(1-|z|^{2}\right)^{k-(n+1)}}{\varphi(z)}\right)^{1 / k} \neq 0
$$

may happen for a suitable pair $p, q$ with $p<q$ even for the simplest weight $\varphi \equiv 1$. To see this, for each $p \in(0, n+1)$ and $q$ sufficiently large, since $1 / k=(1 / p)-(1 / q)$, observe that $k-(n+1)<0$; then

$$
\limsup _{r \rightarrow 1}\left(\frac{\left(1-|z|^{2}\right)^{k-(n+1)}}{\varphi(z)}\right)^{1 / k}=\lim _{r \rightarrow 1}\left(\left(1-|z|^{2}\right)^{k-(n+1)}\right)^{1 / k}=\infty .
$$

Remark 2.5. Of course, in our Theorem 2.3, when $p=q$ the expression

$$
\left(\frac{\left(1-|z|^{2}\right)^{k-(n+1)}}{\varphi(z)}\right)^{1 / k}
$$

should be read as $1-|z|^{2}$.

\section{Acknowledgement}

The author would like to express his thanks to the referee for making him aware of [Ra07]. 


\section{References}

[AC01] A. Aleman and J. Cima, 'An integral operator on $H^{p}$ and Hardy's inequality', J. Anal. Math. 85 (2001), 157-176.

[AS95] A. Aleman and A. G. Siskakis, 'An integral operator on $H^{p}$, Complex Var. 28 (1995), 149-158.

[AS97] A. Aleman and A. G. Siskakis, 'Integration operators on Bergman spaces', Indiana Univ. Math. J. 46 (1997), 337-356.

[Hu03] Z. J. Hu, 'Extended Cesàro operators on mixed norm spaces', Proc. Amer. Math. Soc. 131 (2003), 2171-2179.

[Hu04] Z. J. Hu, 'Extended Cesàro operators on Bergman spaces', J. Math. Anal. Appl. 296 (2004), 435-454.

[HT10] Z. J. Hu and X. M. Tang, 'Schatten(-Herz) class extended Cesàro operators on Bergman spaces in the unit ball of $\mathbb{C}^{n}$, Proc. Amer. Math. Soc. 138 (2010), 2803-2814.

[Ra07] J. Rattya, 'Integration operator acting on Hardy and weighted Bergman spaces', Bull. Aust. Math. Soc. 75 (2007), 431-446.

[Ru80] W. Rudin, Function Theory in the Unit Ball of $\mathbb{C}^{n}$ (Springer, New York, 1980).

[Zh05] K. H. Zhu, Spaces of Holomorphic Functions in the Unit Ball, Graduate Texts in Mathematics, 226 (Springer, New York, 2005).

ZHANGJIAN HU, Department of Mathematics, Huzhou Teachers College, Huzhou, Zhejiang, 313000, China

e-mail: huzj@hutc.zj.cn 